\title{
Las redes de citación como elemento de construcción del campo científico: análisis de la comunicación política en México
}

\section{Alejandra Rodríguez-Estrada ${ }^{1}$}

Recibido: 2017-05-31

Enviado a pares: 2017-06-01
Aprobado por pares: 2017-07-03

Aceptado: 2017-11-09

DOI: 10.5294/pacla.2018.21.3.2

Para citar este artículo / to reference this article / para citar este artigo Rodríguez-Estrada, A. (2018). Las redes de citación como elemento de construcción del campo científico: análisis de la comunicación política en México. Palabra Clave, 21(3), 641-672. DOI: 10.5294/pacla.2018.21.3.2

\section{Resumen}

En el artículo, se comprende la estructuración objetivada del campo científico de la comunicación política en México, a partir de la citación que existe en las publicaciones. Para este propósito, se utilizó la bibliometría y la modelación de sociogramas usando la técnica del análisis de redes sociales (ARS). Se realizó una sistematización documental de escritos de 1970 a 2012, que generó un corpus de 99 publicaciones localizadas en portales de acceso abierto: el Centro de Documentación en Ciencias de la Comunicación del Instituto Tecnológico y de Estudios Superiores de Occidente, la Red de Revistas Científicas de América Latina y el Caribe, España y Portugal, de la Universidad Autónoma del Estado de México, y la base de datos brasileña Scientific Electronic Library Online. El análisis permitió observar, a partir de los datos, un campo relativamente intrincado y activo en las redes de citación, así como la existencia de una dinámica pluridisciplinar cuando se cita a autores externos al campo, sobre todo, del cam-

1 orcid.org/0000-0001-9963-2654. Universidad Autónoma de Puebla, México. ale0323@gmail.com 
po de las ciencias políticas. Se encontró que existe un entramado con una dependencia mutua regular en su estructuración, en especial, por el intercambio de capitales de reconocimiento y relación. El estudio propone una metodología que puede resultar pertinente para estudios comparativos entre países o áreas de conocimiento, en el reconocimiento de la comunicación entre los investigadores y la construcción social del conocimiento.

\section{Palabras clave}

Campo científico; capital científico; comunicación política; cultura de citación; análisis de redes sociales (Fuente: Tesauro de la Unesco). 


\section{Citation Networks as an Element of Construction of the Scientific Field: Analysis of Political Communication in Mexico}

\section{Abstract}

This paper comprises the objectified structure of the scientific field of political communication in Mexico, based on the citations found in published texts. For this purpose, bibliometrics and modeling of sociograms were used using the technique of social network analysis (SNA). A documentary systematization of texts from 1970 to 2012 was carried out, resulting in a corpus of 99 publications found in open-access portals: the Centro de Documentación en Ciencias de la Comunicación del Instituto Tecnológico y de Estudios Superiores de Occidente, the Red de Revistas Científicas de América Latina y el Caribe, España y Portugal of the Autonomous University of Mexico State, and the Brazilian database, Scientific Electronic Library Online. Based on the data found, the analysis showed a relatively intricate and active field in citation networks, as well as the existence of a multidisciplinary dynamic when authors outside the field are cited, especially from the field of political science. Results show that there is a structure of regular mutual dependence, particularly due to the exchange of recognition and relationship capitals. The study proposes a methodology that may be relevant for comparative studies between countries or areas of knowledge in the recognition of communication between researchers and the social construction of knowledge.

\section{Keywords}

Scientific field; scientific capital; political communication; citation culture; social network analysis (Source: Unesco Thesaurus). 


\section{As redes de citação como elemento de construção do campo científico: análise da comunicação política no México}

\section{Resumo}

No artigo, compreende-se a estruturação objetivada do campo científico da comunicação política no México a partir da citação que existe nas publicações. Para esse propósito, utilizou-se a bibliometria e a modelação de sociogramas usando a técnica da análise de redes sociais (ARS). Realizou-se uma sistematização documental de escritos de 1970 a 2012, o que gerou um corpus de 99 publicações encontradas em portais de acesso livre: o Centro de Documentação em Ciências da Comunicação do Instituto Tecnológico e de Estudos Superiores do Ocidente (CC-DOC, ITESO), a Rede de Revistas Científicas da América Latina e Caribe, Espanha e Portugal (Redalyc) da Universidade Autônoma do Estado do México e a base de dados brasileira Scientific Electronic Library Online (SciELO). A análise permitiu observar, a partir dos dados, um campo relativamente intrincado e ativo nas redes de citação, assim como a existência de uma dinâmica pluridisciplinar quando autores externos ao campo são citados, sobretudo do campo das ciências políticas. Descobriu-se que existe uma trama com uma dependência mútua regular na sua estruturação, especialmente pelo intercâmbio de capitais de reconhecimento e relação. $O$ estudo propõe uma metodologia que pode ser pertinente para estudos comparativos entre países ou áreas de conhecimento no reconhecimento da comunicação entre os pesquisadores e a construção social do conhecimento.

\section{Palavras-chave}

Análise de redes sociais; campo científico; capital científico; comunicação política; cultura de citação (Fonte: Tesauro da Unesco). 


\section{Introducción}

La producción científica es la expresión de un trabajo intelectual que se comparte por medios escritos y, en la actualidad, se disemina principalmente por medios electrónicos. Es mediante esa difusión y divulgación que se mueve el conocimiento y se permite la retroalimentación, para legitimar con aceptación o para tensar con crítica los trabajos publicados. La función comunicativa de la ciencia es fundamental para su crecimiento y le da su sentido de ser. Así, la consolidación del trabajo científico, en gran medida, se da por la materialización escrita, las publicaciones, en su mayoría reportadas en revistas científicas, que además siguen un proceso académico de evaluación y reconocimiento. ${ }^{2}$

En este artículo, se plantea la observación del flujo de esos capitales dentro del campo científico de la comunicación política en México. Los resultados expuestos son un ejercicio significativo para plantear y sustentar una propuesta teórico-metodológica, a fin de comprender la citación como una práctica objetivada que genera capital científico en un campo como el de la comunicación política, a fin también de proponer una replicación para otros países y poder establecer comparativos.

Aunque existen algunos reportes sobre la producción científica en México en el tema de la comunicación política, como el estudio que realiza Paláu-Cardona (2009) en su tesis doctoral sobre el campo de la comunicación en la dimensión política, donde plantea un análisis relevante de las publicaciones y de los principales representantes, no existe una expresión sobre la manera en que se concatenan las producciones. Por su parte, Piedra (2009) analiza la producción en el campo de la comunicación en las bases de datos internacionales desde técnicas bibliométricas, a fin de examinar la estructura intelectual del campo durante siete años. Tanto en Chile como en España (Ramos, 2012; Martínez, 2007), hay esfuerzos importantes por comprender cómo se están configurando los campos de conocimiento; sin embargo, en México, ha faltado este esfuerzo por mapear

2 No se toman en cuenta las participaciones de otro tipo, como las orales, ya que no son una práctica objetivada; sin embargo, no quiere decir que no tengan un papel importante en la conformación de las redes y en la circulación científica. 
la comunicación que se articula en la producción científica a partir de la citación (Piedra, 2010).

El interés en tener dicha aproximación se vincula a lo que menciona Martínez (2007), puesto que permite conocer la estructura interna del campo abordando aspectos que ayudan a comprender más a fondo la producción científica.

La ciencia es un escenario que revela intereses, competencias y circunstancias de poder, por ello, comprenderla a la luz del concepto de campo cientifico resulta sugerente, porque permite acercarse a la dimensión de la interacción.

El trabajo científico más consolidado e institucionalizado se produce generalmente en el entorno académico que, por su dinámica, se circunscribe también en contextos políticos y organizacionales que le pueden dar otro sentido a su quehacer. Para revelarlo, el concepto de campo científico de Bourdieu (1997) resulta pertinente, ya que señala qué es la comprensión de fuerzas, luchas de intereses, coaliciones y monopolios, nacionalismos e imperialismos que se generan en entornos desiguales dependientes de la estructura de distribución del capital científico y que, además, a diferencia de otros campos, se produce para los pares que son al tiempo la competencia y así se generan inercias muy cerradas.

Entonces, el concepto de campo cientifico permite comprender interacciones, flujos de poder e intereses, no solo en términos institucionales y organizacionales. Una de las fortalezas del concepto es que involucra la lógica de la interacción a partir del flujo de capitales ${ }^{3}$ y va más allá del concepto un tanto utópico de comunidad científica que incluye la idea de que se buscan objetivos comunes.

3 Desde esa lógica, un capital tiene que ver con la acumulación e intercambio de algo que produce satisfacción. En este caso, se entenderá como acumulación de citas las que otros realicen de la producción científica. Su acumulación por la cantidad de artículos publicados no se disgrega, ya que se entiende como un capital como tal, no importando si es el que tiene más artículos o el que menos, incluso puede pasar que haya quienes escriban más pero no sean tan citados, y viceversa, quienes escriban poco pero sean más citados, así que no es un criterio que se haga proporcional, dado que en su lógica no tendría por qué serlo.

646 Las redes de citación como elemento de construcción del campo científico... - Alejandra Rodríguez-Estrada 
Para tener un acercamiento a la configuración de un campo científico, se debe partir del fenómeno por estudiar, que tiene huellas o representaciones simbólicas que permiten seguir la pista de la manera en que se articulan para dar significado. En la ciencia, son las producciones, los textos, la punta del iceberg que concentra la intención y da luz sobre las interacciones y los encuentros de sentido en un cuerpo de significación científica que tiene como propósito el descubrimiento o la invención.

Los productos científicos suelen ser consecuencia de un entramado, por la socialización con los pares, la exposición a ciertas experiencias y la legitimidad que se les da a ciertos argumentos sobre otros. Dentro del campo circulan capitales que son los que activan dicha dinámica.

La ciencia tiene un poder muy sólido por su pretensión de acercamiento a la verdad y, en la interacción dentro y fuera de la ciencia, hace que haya un debate constante y sostenido. La búsqueda de autoridad científica es un capital acumulable, transmisible y transformable (Bourdieu, 1999), el cual se otorga por el reconocimiento de sus productos. El proceso se genera por un sistema al estilo "meritocrático", donde se obtienen credenciales para ganar autoridad, un reconocimiento socialmente construido y reconstruido.

El capital científico se puede reforzar por prácticas objetivadas o subjetivadas; las primeras se engrosan en función de las evidencias del trabajo y el reporte científico, mientras que las segundas tienen que ver con las apreciaciones del trabajo de cada investigador, es decir, los atributos que se dan a las prácticas objetivadas.

Las prácticas objetivadas en la ciencia se manifiestan en su documentación. Wodak (2003) afirma que son consecuencia de una construcción y confrontación de ideas, donde los textos se convierten en las arenas de combate que tienen las huellas de los discursos y de las ideologías en que se encuentran y que contendieron por su predominio. Por ello, resulta relevante comprender cómo se da esa contienda o intercambio en las publicaciones. 


\section{La citación como práctica objetivada}

El artículo explora, desde la conceptualización que se ha realizado de campo científico, el traslado a una práctica objetivada para soportar el concepto de capital científico. El conocimiento mantiene un discurso vivo y la reproducción de los textos es lo que los mantiene activos (Foucault, 1973). En la ciencia, esto se relaciona con el reconocimiento entre pares, que suele ser recurrente en la literatura que aborda la producción científica. Una forma de reconocer al par es citándolo o colaborando con él, así se explica su sentido de ser y hacer ciencia. Los textos y la vinculación de unos con otros por la citación son una de las maneras objetivas de observar la producción científica y su estructuración.

Así es como la cita que hace alguien más del trabajo de un par se vuelve un capital científico. Se cumple la función de "enlace argumentativo y de apoyo empírico con otros textos” (Ramos, 2012, p. 9) que permite que la producción científica se vaya consolidando en el campo.

Las citas se convierten en indicadores de conexiones sociales dentro del campo, de alianzas y de estrategias que permiten observar las conexiones de la dinámica del campo, desde sus sistemas de comunicación; el entramado, entonces, permite conocer tanto la dimensión social como la cognitiva de la ciencia (Ramos, 2012). Incluso, no importa si se hace una cita para desmentir o criticar, es en la ruptura de paradigmas donde se produce ese proceso científico. Así que, o para reforzar o para refutar, la discusión o el diálogo es el elemento indispensable para construir ciencia.

Entonces, el capital científico se pone en juego y los productores dominantes consiguen imponer la definición de ciencia o de lo que es científico, "en la cual su realización más acabada consiste en tener, ser y hacer, lo que ellos tienen, son y hacen” (Bourdieu, 1999, p. 20). Es decir, se le concede la legitimidad como autoridad en ciencia, y convertirse en lo que coloquialmente se dice: ser una institución en sí mismos.

Sin embargo, no todos coinciden en que ocurra de esa manera. Por su parte, Romancini (2006) afirma que existen situaciones como el "efecto 
Mateus" que genera una distorsión a la atribución de estatus representado por el reconocimiento medido por citación; un estudio puede ser relevante e innovador, pero pueden pasar muchos años sin recibir citas; y por otro lado, hay documentos que pueden recibir muchas citas solo para impresionar o demostrar lealtades a un actor en determinada teoría.

Moody (2004), por su parte, ha sugerido que la producción de un conjunto de ideas generadas por los científicos está en función del grupo de personas con las que interactúan. Los científicos suelen intercambiar ideas, técnicas de trabajo e influirse a partir de las redes de colaboración, pero dichas redes no han sido del todo exploradas.

Además, hay pocos trabajos que pretenden ver cómo las interacciones entre distintas disciplinas, con un objeto de estudio similar, pueden dar lugar a un campo de conocimiento (Moody, 2004); para el caso de este proyecto, la comunicación política puede tener colaboradores desde la sociología, la psicología, la antropología, la comunicación y las ciencias políticas. El estudio de la comunicación política, además, plantea la posibilidad de observar cómo se da un producto socialmente construido y cómo a veces puede existir la pretensión de ser el conocimiento de alguna realidad (Olivé, 1988). Tener un acercamiento a la manera en que se estructuran los intercambios y comprender cómo se da socialmente esa construcción resulta relevante.

\section{El campo científico de la comunicación política en México}

Una vez explicado el marco teórico que fundamente el análisis, se pretende estudiar con esos lentes un campo que por su objeto de estudio y por las características resulta relevante. Aunque el propósito de este estudio no es profundizar en la comunicación política en sí, en su interior cognitivo, sin embargo, se deben entender algunos mecanismos propios de su lógica de estructuración histórica y las circunstancias discursivas en las que se ha ido configurando, a fin de identificar tendencias y rupturas.

La historia de la comunicación política se comprende a partir de necesidades teóricas y prácticas de los contextos en los que se ha generado. 
Los antecedentes de la comunicación política se vinculan al mismo proceso del desarrollo teórico de la comunicación. Por ejemplo, el desarrollo de la comunicación en los Estados Unidos inicia por la inquietud de conocer procesos de opinión pública, difusión y propaganda. Los principales representantes entre las décadas de 1920 y 1940 son Harold Lasswell, Paul Lazarsfeld y Kurt Lewin. Los tres con perfiles académicos distintos, pero con aportes fundamentales al campo de la comunicación y propaganda.

Se conciben, entonces, las primeras teorías de comunicación y se despierta gran interés en el tema, sobre todo, por el contexto electoral norteamericano y sus necesidades prácticas de persuasión y, eventualmente, la medición de dicha persuasión. En la década de 1940, se hace un estudio sobre las elecciones presidenciales del país, llamado People’s Choice, con la intención de conocer las motivaciones en la decisión de voto, estudio realizado por Lasarzfeld, Berelson y Gaudet (1944) en la Universidad de Columbia. Esa corriente de estudios se volvió el paradigma de los estudios de la comunicación política (Martins, 2007), sobre todo, en la corriente anglosajona ${ }^{4}$.

Por el contrario, en América Latina se presenta una influencia, en especial, de la corriente europea, con un enfoque teórico muy distinto del de la corriente norteamericana. Dadas las circunstancias históricas de posguerra, la corriente europea tiene un corte crítico, cuestionador del papel del Estado y de la función que cumplen los medios masivos en la cultura y el poder (Mattelart, 1997). Este tipo de estudios ubica como punto de partida la estructura de poder de los medios y sus características como aparatos

4 La forma en que se genera la teoría de la comunicación en Norteamérica llama la atención, pues se presenta, primero, una observación a fenómenos propios de la comunicación política, para de ahí expandirse al estudio de la comunicación en general. Podría pensarse que, primero, se dieron las teorías de la comunicación, y de ahí las de la comunicación política que comprende a una como derivada de la otra. En la dinámica de desarrollo del conocimiento norteamericano, se dio a la inversa. Además, podría decirse que, desde 1920, el empirismo ha sido la base de la construcción social del conocimiento en la corriente norteamericana de la comunicación. La Escuela de Chicago es su principal referente. Ya hacia la década de 1940, la siguiente corriente es la Mass Communication Research, que se centra en el análisis funcional. En 1937, se forma la American Association for Public Opinion Research que hace la primera publicación académica sobre comunicación masiva Public Opinion Quarterly (Mattelart, 1997) y, que como su nombre lo dice, está referida a la opinión pública y, por tanto, está vinculada al fenómeno de la comunicación política. 
ideológicos del Estado moderno, con enfoques particularmente de corte marxistas con cuatro corrientes: "La escuela crítica de Frankfurt, la economía política, el imperialismo cultural y los estudios culturales" (León, 2002, p. 8), abanderados por teóricos destacados como Luis Ramiro Beltrán, Enrique Sánchez Ruiz y Armand Mattelart. Aunque no se puede dejar de lado que también existe la corriente de investigación norteamericana que es más de corte instrumental (León, 2002).

Desde esa línea de argumentación se desarrolla una corriente de pensamiento crítico en la Escuela de Frankfurt y se describe por primera vez la lógica industrial de la cultura, con sus riesgos de uniformidad y distracción conformista para el ciudadano/consumidor de mensajes masivos ( $\mathrm{Pi}$ neda, 2001). Así pues, el discurso del poder y su crítica está presente en la corriente europea de raíz marxista que describe un complejo campo cultural que, a través de la industria y los medios, permea a la sociedad, según los intereses de una clase hegemónica y elitista, de acuerdo con sus postulados.

La naturaleza de la vertiente europea lleva a América Latina a desarrollar sus propias reflexiones en contextos, sobre todo, de atraso y, al mismo tiempo, de imperialismo cultural. Europeos como Armand Mattelart trasladan los estudios críticos a los contextos desiguales de la región, lo cuales propician que se genere un conocimiento propio. En este sentido, se produce una corriente de pensamiento que origina un ejercicio, en especial, de tipo ensayístico y filosófico, frente al tema de medios y poder en sociedades proclamadas como democráticas y que se enmarcan por prácticas comunicativas desiguales.

Específicamente en México, antes de la década de 1980, los empiristas de la comunicación política trabajaban en investigación aplicada o por encargo para fines comerciales o políticos. En esta década, se presentó una crisis en el campo de la comunicación en general: se cerraron algunos centros de investigación y hubo una disminución hasta de $50 \%$ en los apoyos y en los salarios de los académicos (Fuentes-Navarro, 1998). De acuerdo con este autor, es en la década de 1990 que el campo de la comunicación empieza a legitimarse a partir de los procesos de institucionalización y 
profesionalización. Ese panorama histórico permite ubicar las primeras configuraciones en el campo.

Hay campos científicos que han sido mayormente reflexionados, pero existen otros nuevos y poco estudiados. Por ejemplo, en la revisión de la literatura, se encontró que en el campo de la medicina hay muchos estudios y reflexión de sí misma (Sogi, Perales, Anderson y Bravo, 2002). Otros campos, por la propia juventud de su existencia, han sido menos estudiados, como el campo de la comunicación en México, que es bastante reciente; su existencia se aproxima a la década de 1970, de acuerdo con los estudios de Paláu-Cardona (2009) y Fuentes-Navarro (1998). Formalmente, dichos estudios surgieron en la década de 1908, y su débil consolidación se manifiesta, por ejemplo, en que en la indización del Consejo Nacional de Ciencia y Tecnología (Conacyt) solo hay una revista del área "Comunicación y Sociedad" de la Universidad de Guadalajara. Las peculiaridades de un campo en consolidación hacen que las interacciones con otros campos o disciplinas también puedan tener un papel importante en su constitución.

Actualmente, se ha puesto mayor atención en los sistemas y procesos de comunicación que se dan en los debates públicos, lo cual ha propiciado que los agentes sociales especializados en la operación e investigación científica de ellos intervengan más en la producción de estos procesos (Fuentes-Navarro, 1998). Tal fenómeno lleva a cuestionar el quehacer científico de esta área de investigación, dado que sus formas de intervenir y comunicar pueden ser consecuencia de la legitimación que se fortalece por las redes de pares en que se encuentren incorporadas.

En México, se han presentado acontecimientos políticos que han propiciado procesos de opinión pública que dan pie a la reconfiguración o el reforzamiento de un establishment político, social y cultural. Algunas áreas de conocimiento tienen como objeto de estudio conocer estos fenómenos. En este sentido, la comunicación política, como se ha dicho, estudia cómo se da el intercambio de información entre los gobernantes y los gobernados con la finalidad de conocer cómo son los procesos de 
comunicación entre los que hacen la política y los que reciben el servicio de los políticos (Ochoa, 2000; Reyes, 2007). Se trata de describir una interacción que permite el funcionamiento de una sociedad que se proclama democrática.

En cuanto a la producción científica en comunicación política, Aceves (2002) afirma que antes de 1988 se habían realizado solo dos trabajos de investigación en el tema de medios y política, y que, de esa fecha en adelante, hay más de sesenta trabajos publicados. La atención en los fenómenos de comunicación política tiene más incidencia por parte de investigadores que publican en revistas internacionales. En México, ya en las últimas dos décadas, los estudios de medios tienden a abordar mayormente el aspecto político de los procesos de producción de la comunicación (Paláu-Cardona, 2009, p. 68).

En el análisis documental que realizó Fuentes-Navarro (1995) a 686 documentos de comunicación, 140 de ellos tienen un predominio temático sobre la política. Lo que reporta que los temas relativos a la comunicación política son áreas de alta producción en comunicación.

Una de las cualidades que hacen que este estudio se interese en el campo de la comunicación política, y no en otro, tiene que ver con su posibilidad interdisciplinar; al tener confluencia de distintas disciplinas, pareciera ser un campo rico y nutrido por el debate e intercambio de ideas y de aproximaciones.

\section{Diseño metodológico}

Para acercarse a la conformación del campo científico de la comunicación política en México, se realizó un análisis a la producción científica por la citación entre los investigadores. Para el análisis de las publicaciones, se hizo una sistematización documental de escritos de $1970^{5}$ a 2012, a fin de tomar en el rango a 1988, que es cuando se detona el debate en torno a la posibilidad del cambio en el poder y se discute el manejo de información de los

5 Aceves (2002) aseguraba que los estudios antes de 1988 eran menos que los que se produjeron posteriormente; por ello, decidió tomarse una década previa, además de considerar su disponibilidad en las bases de datos electrónicas. 
medios en México. Además, se considera un tiempo que permite conocer la producción de conocimiento previo y posterior a una coyuntura política, que empieza a plantear la posibilidad de "la alternancia política en México" (Ojeda, 2004) y que, probablemente, esboza nuevos esquemas políticos y sociales y, por tanto, el replanteamiento en algunas de las producciones del campo de la comunicación política. La unidad de análisis de las publicaciones será la cita en estas, que podrían ser de revistas mexicanas o latinoamericanas, de comunicólogos, politólogos o psicólogos, de autores mexicanos o extranjeros. El criterio fue que abordaran temas de comunicación política en México. Ya que la configuración del campo no debe ser solo de tipo disciplinar, es decir, la construcción de conocimiento sobre comunicación política va más allá de la circunscripción disciplinar y la comprensión científica, también tiene que abrirse y analizarse por otros mecanismos, sobre todo, de interacción con el conocimiento, como es el caso de la citación.

Para el análisis, se hizo una la búsqueda electrónica de textos relacionados con la comunicación política en México. Las plataformas para obtener los textos fueron el Centro de Documentación en Ciencias de la Comunicación ${ }^{6}$ del Instituto Tecnológico y de Estudios Superiores de Occidente, la Red de Revistas Científicas de América Latina y el Caribe, España y Portugal, de la Universidad Autónoma del Estado de México, y la base de datos brasileña Scientific Electronic Library Online. ${ }^{7}$ Se parte del criterio de que los artículos obtenidos de estas bases de datos tienen más posibilidades de difusión y divulgación por su comunicabilidad electrónica y acceso gratuito, y así se incrementa la posibilidad de citación.

En el campo de búsqueda, se pusieron las palabras clave: comunicación política, comunicación y democracia, comunicación y gobierno, opinión pública, medios y política, medios y democracia, medios y gobierno, y otros conceptos similares, todos en español.

\footnotetext{
6 Se eligió a las bases de datos de habla hispana y de acceso abierto, ya que se considera que eso permite una mayor circulación de ideas, sin importar las condiciones estructurales de las instituciones donde trabajen los investigadores latinoamericanos.

7 En https://goo.gl/1Sxwij con el título de anexo I se puede buscar el listado de revistas consultadas.
} 
Otra herramienta que resulta útil para el análisis del campo científico es el conocimiento de las redes de colaboración por citación entre los investigadores, para lo cual el trabajo se apoyó en la herramienta metodológica análisis de redes sociales (ARS) que se utiliza para comprender el estudio de la estructura de las relaciones entre diversas entidades, apoyada en la formalización matemática que permite el uso de gráficas para la modelación y el análisis de distintas redes (Gil y Ruiz, 2009), además el paradigma del análisis de redes sociales es una aproximación muy pertinente para comprender la coautoría y cocitación en las publicaciones académicas (Kuzhabekova, 2011).

El análisis de redes sociales ayuda a entender, de manera conjunta con el análisis bibliométrico, las redes de citación en las publicaciones sobre comunicación política. En la literatura norteamericana, sobre todo, es una herramienta metodológica recurrente para observar la colaboración entre los científicos. Al respecto, se han publicado artículos que afirman que es un recurso que visualiza el panorama de colaboración (Gaete y Vásquez, 2008; Gil y Ruiz, 2009).

Estas herramientas ratifican conocer el panorama tanto material como objetivo del campo y dan una visión de su constitución y de la forma en que se producen los textos entre los autores de diversas disciplinas. También a estos estudios se les conoce como prácticas de difusión, lo cual permite conocer los procesos de colaboración y socialización del conocimiento en la ciencia (Quatman y Chelladruai, 2006).

Ahora bien, en los resultados se presentan sociogramas ${ }^{8}$ que ilustran la manera en que se dan las vinculaciones entre los actores (nodos) y las relaciones (lazos) de unos con otros. Las matrices dan lugar a dichos sociogramas y permiten vaciar las relaciones que hay entre dos elementos a partir de unidades que vinculan los datos de la fila con los de la columna (tabla 1) y contabilizar por este medio la intensidad de la relación.

8 No se incluyen sociogramas para ilustrar, por el espacio para el artículo; sin embargo, más adelante en el análisis se puede observar su diagramado. 


\section{Tabla 1. Ejemplo de matriz que representa cocitación de autores}

\begin{tabular}{|l|c|c|c|c|}
\hline & Aceves & Sánchez Ruiz & Orozco & Calles \\
\hline Francisco Aceves & 19 & 3 & 0 & 2 \\
\hline Enrique Sánchez Ruiz & 1 & 62 & 13 & 0 \\
\hline Guillermo Orozco & 0 & 1 & 2 & 0 \\
\hline Jorge Calles & 0 & 0 & 2 & 0 \\
\hline
\end{tabular}

Fuente: elaboración propia.

A partir de las bases de datos generadas y los artículos recabados, se elaboró la matriz de cocitación, para la cual se siguió el siguiente criterio de análisis: el corpus se conformó por los textos encontrados en los buscadores ya mencionados, con un total de 99 documentos, de los que se disponía del texto completo en español. En la matriz ${ }^{9}$ de cocitación (matriz1), ${ }^{10}$ se pretende encontrar la dinámica entre los autores para sustentar sus argumentaciones. Lo que permite observar el entretejido del campo y, a su vez, el reconocimiento por los aportes de los autores en las ideas, ejemplificaciones o maneras metodológicas de abordar el tema de la comunicación política en México.

Se construyó una red direccional y no simétrica de investigadores, la matriz binaria (matriz1), compuesta en la fila por los autores de los documentos, es decir, los autores citantes de otros investigadores, con $74,{ }^{11}$ y las columnas se fueron llenando por los autores citados con 155; se contabilizaron en total 1013 citas. La red se articuló por 228 nodos y 338 relacio-

9 El procedimiento de captura consistió, en primer lugar, en ir al listado de referencias bibliográficas e identificar a los autores y, posteriormente, se hizo la búsqueda en el texto y se contaron las veces que apareciera el nombre del autor o la referencia; no se contabilizó la aparición en la bibliografía.

10 Las matrices completas se pueden consultar en el sito bajo el título de análisis de redes sociales https://goo.gl/ ZqN8wx. Para este estudio, se reportan dos sociogramas (matriz2 y matriz3) por cuestiones de espacio, además son las que permiten explicar de mejor manera el entramado del campo.

11 Se analizaron 99 documentos, algunos de los cuales eran del mismo autor, pero de distinto contenido; por ello, se tiene el registro en fila de 73 autores. Además, se considera el criterio de la concentración de citas por autor como parte de su capital científico, es decir, puede tener mucha o poca producción y no necesariamente mucha citación, pues eso dependerá de los demás investigadores. 
nes entre ellos y, para el sociograma y el cálculo de medidas estructurales, se utilizó UCINET $6 .^{12}$

\section{Figura 1. Características y composición de matrices}
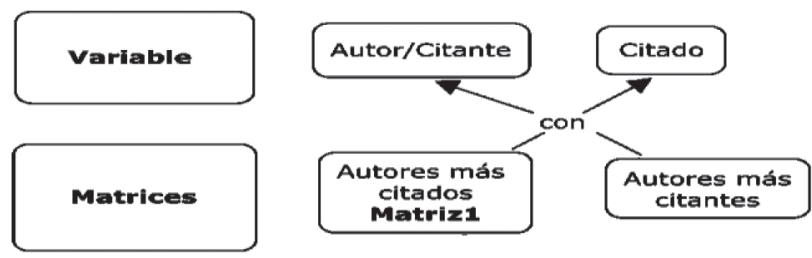

Correspondencia

Matrizz

Fuente: elaboración propia.

De la matriz1 se extrajo la matriz2 y, en esta otra, se tomaron solo los investigadores que tenían más actividad dentro de matriz1, a fin de tener mayor densidad y observar el fenómeno en su sustancia concentrada, discriminando aquellos que tenían menos de cinco citas, que en estudios como el de Rodríguez-Estrada (2015) se observa como la tendencia de citación entre los investigadores del campo de la comunicación política en México (figura 1).

De la matriz1 también se extrajo otra matriz simétrica más pequeña (matriz3), a fin de poder observar la correspondencia de un autor con otro, además de esta solo se dejaron los autores de la columna que hayan sido citados por otro de ella, y se eliminaron a los que en la fila no fueron nombrados por los de la columna que sumaron 35 autores citantes (fila) y 35 citados (columna), y en total se contaron 432 citas de esta matriz.

En todas las matrices, se calcula la densidad, pues es esa propiedad estructural que permite conocer la proporción de vínculos posibles que se pueden dar en una red (Van den Bulte y Wuyts, 2007). La forma de calcularla es a partir de la división del número de vínculos que hay en la red entre el número total de vínculos posibles de ella. Esto sirve para conocer el porcentaje de densidad de la red para cada matriz, lo cual nos permite conocer la posibilidad de citación o cocitación.

12 Los diagramas tienen una finalidad de tipo ilustrativo, sobre todo, para visualizar el entramado de la red. 
Para tener un acercamiento a la idea de productividad de un científico, se considerará el número de citaciones de "la posición que ocupa un autor con respecto a otros como posición de poder, prestigio" (Ramírez, 2010, p. 27), que dentro de la lógica del campo es parte de la lucha de poder. También se trata de dilucidar los patrones y comportamientos de citación que se experimentan en un campo determinado.

Se usaron otras medidas de centralidad que ayudan a identificar la posición de los actores en la red. El degree indica el número de relaciones que tiene un individuo, es la variación en los grados de los vértices dividido por el máximo grado de variación, el cual es posible en una red del mismo tamaño; y closeness indica qué tan cerca está un individuo de otros actores en la red (Van den Bulte y Wuyts, 2007).

El closeness centrality permite conocer cómo está distribuida la red de investigación, sobre todo, por grupos de investigación, y distinguir en los nodos quiénes son aquellos que están más cerca de otros actores de la red. Con estas funciones, se puede conocer más sobre la constitución de aquella entre investigadores, tanto en lo que están reportando como en lo que está objetivado en sus publicaciones.

Recursos como la bibliometría y el análisis de redes sociales dan una aproximación cuantitativa de un intercambio de capitales científicos, sobre todo, relacionado con el capital de autoridad, como se verá ilustrado en los siguientes resultados del campo de la comunicación política en México.

\section{Resultados}

El análisis bibliométrico permite tener una "dimensión cognoscitiva de la producción: que tiene que ver con el establecimiento del formato artículo como artefacto para hacer circular las propuestas y resultados de investigación" (Fuentes-Navarro, 1995, p. 255). Y, a partir de dicho artefacto, las citas se convierten en una especie de evidencia del entretejido de la construcción de conocimiento y del diálogo entre sus actores.

Como se ha mencionado, los mecanismos formales son parte de la estructura institucionalizada. A través de la citación, se puede tener una 
aproximación a la manera objetivada en que los investigadores dialogan en sus producciones.

\section{Citación como práctica objetivada del campo científico de la comunicación política}

La citación permite conocer de manera objetivada los intercambios en la producción científica y, así, se consolida el capital científico desde su socialización intelectual. Las citas contribuyen al capital de autoridad cuando su argumentación se legitima en los textos de otros, se vuelven un capital relacional por ponerse en diálogo con las ideas de quien lo cita y, por tanto, se suman al capital científico de cada investigador. Este capital científico se acumulará para cada investigador en función de las citas que se hagan de él. En este primer plano, el conteo de citaciones de cada uno permite observar principalmente su capital de autoridad individual.

En la tabla 2, podemos observar los autores más citados en los documentos que se vertieron en la matriz1. El listado corresponde con lo enunciado en estudios similares, como el de Fuentes-Navarro (1995) y Paláu-Cardona (2009), que, aunque en tiempos muy distintos, y con enfoques de investigación diferentes, reflejan una consistencia en quienes producen con más autoridad académica y visibilidad pública en el campo de la comunicación en México.

Los investigadores con mayor trayectoria y productividad tenderán a ser los más citados. En la tesis de Fuentes-Navarro (1995, p. 253), hay un listado de 25 investigadores con mayor número de productos publicados de comunicación en México de 1986-1994. Se puede observar, al ver los datos de su trabajo y los de la tabla 2, una coincidencia de seis investigadores.

Por su parte, Paláu-Cardona (2009) hizo un listado de 16 investigadores, los más citados en textos publicados por la Asociación Mexicana de Investigadores de la Comunicación y el Consejo Nacional de Educación en Investigación en Ciencias de la Comunicación entre 2001 y 2006; la coincidencia fue menor, solo de cuatro investigadores, pero se puede observar que son cuatro de ellos que también fueron señalados por su director de 
tesis Raúl Fuentes, y los dos primeros Raúl Trejo Delarbre y Enrique Ernesto Sánchez Ruiz en ambos listados están presentes.

\section{Tabla 2. Número de citas a autores en comunicación política}

\begin{tabular}{|l|c|c|c|}
\hline \multicolumn{1}{|c|}{ Citas } & Citas & Autocitas & Total de citas \\
\hline${ }^{* *}$ Raúl Trejo Delarbre & 99 & 2 & 101 \\
\hline${ }^{*}$ Guillermo Orozco & 52 & 2 & 54 \\
\hline Rafael Segovia & 32 & 0 & 32 \\
\hline María Antonieta Rebeil & 28 & 0 & 28 \\
\hline Pablo González Casanova & 24 & 0 & 24 \\
\hline *Pablo Arredondo & 23 & 1 & 24 \\
\hline Alberto Montoya & 23 & 0 & 23 \\
\hline **Enrique Sánchez Ruiz & 21 & 62 & 83 \\
\hline *Francisco Aceves & 20 & 19 & 39 \\
\hline Manuel Antonio Garretón & 20 & 0 & 20 \\
\hline Germán Espino Sánchez & 16 & 12 & 28 \\
\hline José Carlos Lozano & 15 & 0 & 15 \\
\hline
\end{tabular}

*El asterisco en el nombre tiene que ver con la coincidencia con el listado de Fuentes-Navarro (1995).

**Dos asteriscos significa que también coincide con el listado de Paláu-Cardona (2009).

Nota: las autocitas no son representativas en la mayoría de los casos, sin embargo, se reportan con la finalidad de mostrar también un comportamiento de comunicación propia de los autores del campo.

Fuente: elaboración propia.

Estos datos nos permiten situar a los investigadores más consolidados teóricamente, lo cual vislumbra parte de su estructuración. Además, se puede observar cómo la práctica objetivada de citación se transforma en un capital de autoridad científica para aquellos investigadores que tienen más citas.

Los cinco investigadores con más citas de otros: Raúl Trejo Delarbre (99), Guillermo Orozco (52), Rafael Segovia (32), María Antonieta Rebeil (28) y Pablo González Casanova (24), no tienen tanto en común como podría suponerse. Los dos primeros, así como María Antonieta, tienen amplia publicación en el campo de la comunicación en México. Otros investigadores no pertenecen al campo, Rafael Segovia es politólogo y no escribe para este ámbito, no obstante, pudiera ser un hito cuando se quiere 
hablar de cuestiones políticas, así como ocurre con Pablo González Casanova, cuando se hace referencia a la democracia en México.

Es decir, se percibe, por el ejercicio de quienes los citan a ellos, que hay una interacción con el fin de construir conocimiento interdisciplinarmente cuando se necesita explicar desde la comunicación o desde las ciencias políticas el fenómeno abordado. Por ello, es posible que sean más citados aquellos que hablan de temas como la democracia y la participación política en México. En este primer momento, se puede hablar de un principio de heterofilia ${ }^{13}$ entre los pares citados que forman parte del campo (Rogers, 2003); es decir, entre ellos no hay tantas similitudes, sobre todo, por su perfil académico.

El otro comportamiento de citación se observa en la tabla 3, aquellos autores que más citan a otros autores dentro de sus trabajos, es decir, qué investigadores son los que más recurren a citar a sus pares dentro de sus productos. Las citas van de un rango de las 264 a las cuatro por autor. Cabe señalar que es el total de citas que se encontraron de las 99 publicaciones analizadas, por lo cual es posible que sean citas en más de una publicación por autor.

Estos resultados dan muestra de aquellos autores que, en sus producciones científicas, entretejieron argumentación con otros pares, y podrían señalarse que son los que en esa práctica están participando en la construcción de dicho campo, al dialogar con los autores y propiciar el capital relacional. En estos resultados, vemos la coincidencia con algunos autores de los más citados.

De la matriz general de citación (matriz1) se extrajo una matriz de los más citados (matriz2). Con la finalidad de aproximarnos más a la dinámica de citado-citante, en esta otra matriz se eliminaron los autores que tenían menos de cinco citas. Por ello, en su medida de centralidad, podemos

13 En la naturaleza del flujo de comunicación en las redes interpersonales, se puede observar el concepto de homofilia o heterofilia. El principio de homofilia se refiere al grado en el cual un conjunto de individuos que se comunican es similar, mientras que en la heterofilia sucede a la inversa, suelen ser distintos en sus atributos, que pueden ser en creencias, educación, intereses, etc. (Rogers, 2003). 


\section{Tabla 3. Autores que hicieron más citas de otros autores en las publicaciones analizadas}

\begin{tabular}{|l|c|c|c|}
\hline \multicolumn{1}{|c|}{ Autor citante } & Citas & Autocitas & Total de citas \\
\hline Aimé Vega & 264 & 4 & 268 \\
\hline *Enrique Sánchez Ruiz & 116 & 62 & 178 \\
\hline *Francisco Aceves & 97 & 19 & 106 \\
\hline Salvador de León & 37 & 0 & 37 \\
\hline Luis Fernando Sánchez Murillo & 33 & 1 & 34 \\
\hline José Carreño Carlón & 26 & 0 & 26 \\
\hline Rebeca Domínguez Cortina & 25 & 1 & 26 \\
\hline Armando Ibarra & 22 & 0 & 22 \\
\hline *Pablo Arredondo & 18 & 1 & 19 \\
\hline Alejandro Byrd Orozco & 15 & 0 & 15 \\
\hline Norma Pareja & 12 & 2 & 14 \\
\hline
\end{tabular}

*El asterisco en el nombre tiene que ver con la coincidencia con el listado de Fuentes-Navarro (1995).

Fuente: elaboración propia.

ver una densidad de $32 \%$, mientras que en la matriz1 se tuvo una densidad de $8 \%$, dado que se ubicaron 22 nodos insolados, ${ }^{14}$ es decir que no establecieron relación con los demás autores. En esta otra matriz2, se hace un enfoque en el cluster central de la red general. Podemos observar una red más sólida en el intercambio de argumentos para la generación de conocimiento en comunicación política. Y lo que se ilustra es un diálogo de citación moderado, pero relativamente sólido.

A partir del sociograma de la figura 2, podemos ver que en el efecto de citar a otro se propicia una red que permite más interacciones, los puntos representan a autores que citan. Así, la autora Aimé Vega (degree de 108, incloseness de 5.3, outcloseness de 7.4) es la que realiza más trabajo de citación, por lo cual sería central en el efecto de citar, y Raúl Trejo Delarbre (degree de 87 , incloseness de 9.7 y outcloseness de 2.9 ) es central por ser el investigador más citado.

De la figura 2 también podemos ver a quienes concentran mayor interacción de citación. De los que suelen citar más se encontró, además de

14 Esto suele ocurrir cuando existen autocitas. 


\section{Figura 2. Sociograma de autores que más citan a otros autores}

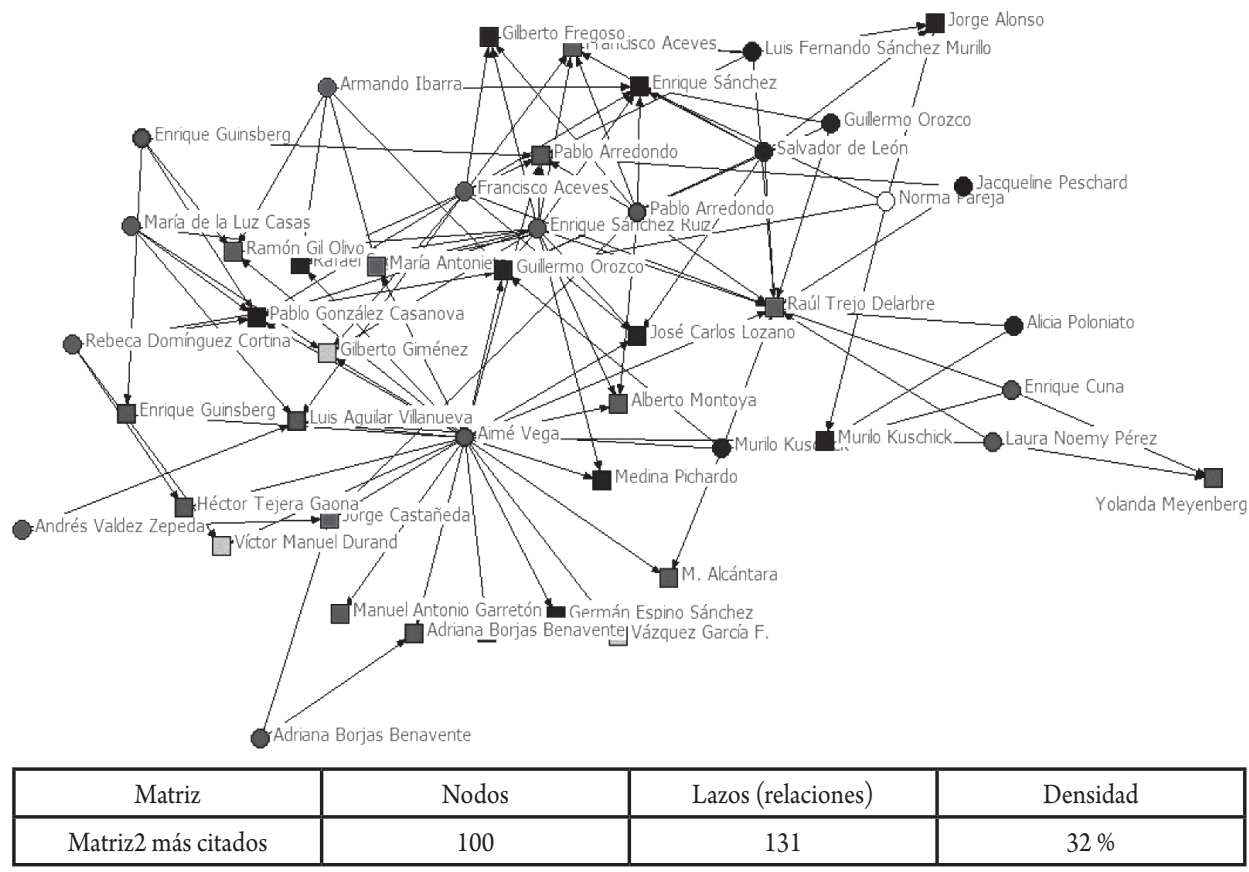

Nota: la densidad se pone en porcentaje, donde 1 = $100 \%$, que significaría en ese caso que todos los nodos están conectados.

Fuente: elaboración propia.

Aimé Vega, a Francisco Aceves, Enrique Sánchez Ruiz y Luis Fernando Sánchez Murillo. Mientras que los más citados fueron, además de Raúl Trejo, Enrique Sánchez Ruiz, Guillermo Orozco, Germán Espino y Pablo Arredondo. Respecto de la interacción, entonces, podemos destacar a Francisco Aceces y Enrique Sánchez Ruiz como los que citan más y son más citados.

Aquellos que reciben capital científico por ser citados por otros parecieran entender la importancia de dicho capital y también suelen citar en sus trabajos a los colegas que pone en juego una generación de conocimiento más dinámica. Desde la otra perspectiva, es decir, los que menos citan a otros, también suelen ser menos citados, lo cual pareciera indicar que aquellos que se mantienen aislados de la producción de conocimiento por la interacción por citas están más lejanos del capital de reconocimiento. Esta dinámica habla de una intercitación relativamente fuerte. 
Hay autores que tienen un estilo de citación de acuerdo con la formación que recibieron. Algunos autores suelen citar más a autores extranjeros, ya que se formaron en otros países, sin embargo, no necesariamente en todos los casos, como se ha visto en autores como Enrique Sánchez Ruiz que realizó su doctorado en la Universidad de Stanford y no sigue esa tendencia. No obstante, hay otros investigadores que sí tienen ese modelo de citación.

A fin de comprender más el fenómeno de correspondencia entre ser citado y citar, se corrió una matriz donde solo se tuviera una sola entrada (matriz3), es decir, los que citan estarían en columna; y los mismos, en fila. Haciendo una matriz simétrica de 35 autores, esta presentó una densidad de $25 \%$.

Figura 3. Sociograma de la matriz correspondencia de autores

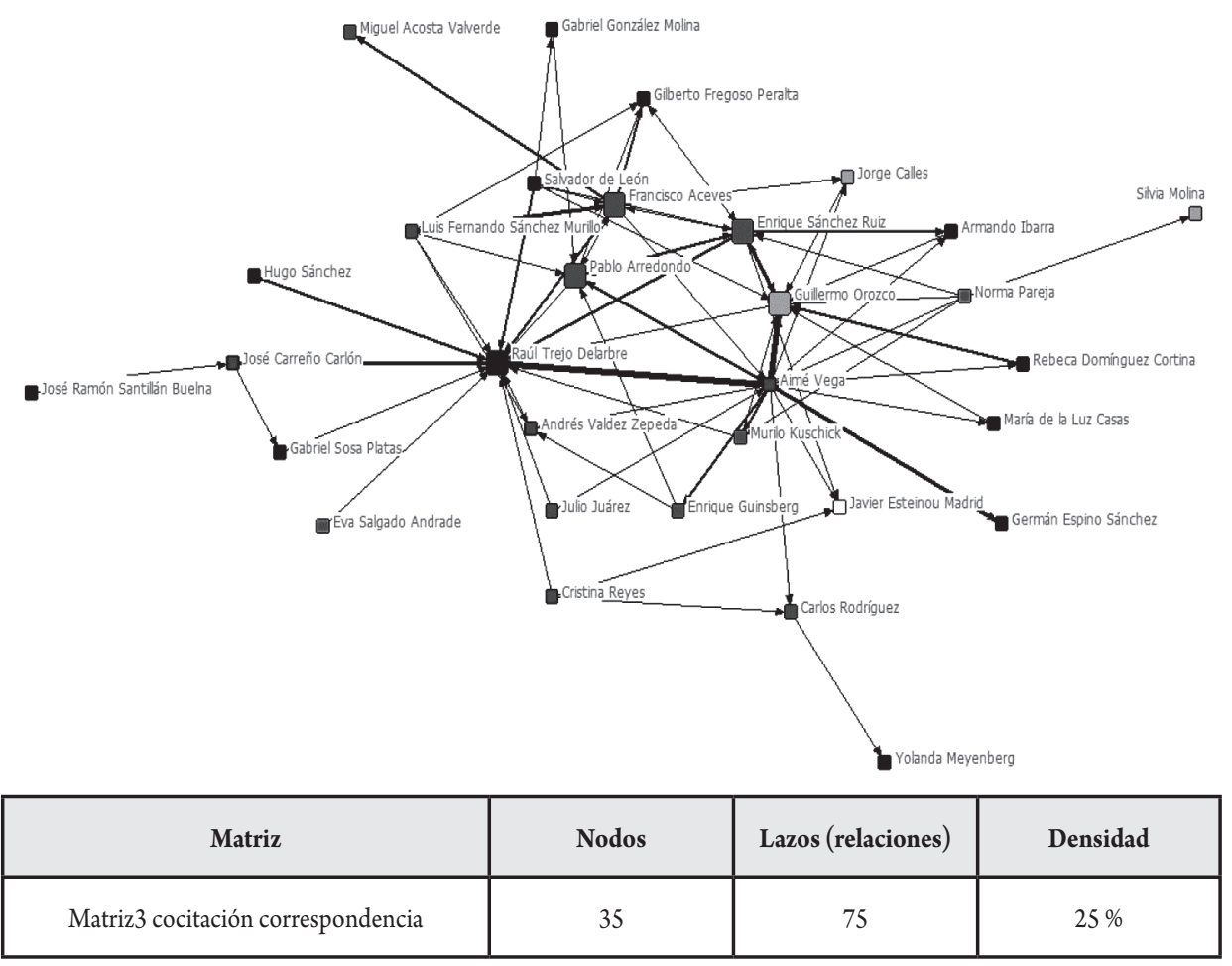

Nota: la densidad se pone en porcentaje, donde 1 = $100 \%$, que significaría en ese caso que todos los nodos están conectados.

Fuente: elaboración propia. 
En el sociograma de correspondencia (figura 3), se puede observar a los autores que citan más o son más citados dependiendo de la flecha; si el vínculo es más grueso, quiere decir que el autor es más citado por ese otro autor, o viceversa. Se trata de una matriz de una sola entrada, es decir, los mismos que están en columna están en fila, y no es de doble entrada como la anterior.

El listado de los investigadores más citados (tabla 2) ofrece una primera panorámica de aquellos autores que están más “activos" en los textos de sus pares. Su presencia intelectual da una visión del campo y las interacciones por citación permiten imaginar la densidad del diálogo en la construcción de conocimientos.

Con estos resultados, se puede ir observando una cultura de la citación; el comportamiento de citar suele ser distinto en cada área de conocimiento o en cada país. Por ello, vale la pena conocer la manera en que los autores suelen citar los trabajos de otros, es decir, qué tanto suele citar un investigador el trabajo de otros. En la tabla 4, se registraron por rangos las cantidades de citas que se suelen hacer entre autores.

\section{Tabla 4. Tendencia de citación sobre comunicación política}

\begin{tabular}{|l|c|c|c|}
\hline \multicolumn{1}{|c|}{ Rango de citación } & Frecuencia & Porcentaje & Citas totales $^{*}$ \\
\hline 0 citas & 14 & 19 & 0 \\
\hline De 1 a 5 & 25 & 34 & 54 \\
\hline De 6 a 10 & 16 & 22 & 127 \\
\hline De 11 a 15 & 8 & 11 & 100 \\
\hline De 16 a 20 & 2 & 3 & 35 \\
\hline De 21 a 30 & 3 & 4 & 74 \\
\hline De 31 a 55 & 2 & 3 & 71 \\
\hline De 56 a 268 citas & 3 & 4 & 552 \\
\hline Total & 73 & 100 & 1013 \\
\hline
\end{tabular}

${ }^{*}$ Cantidad total de citas en la matriz1, por ejemplo se contabilizó que 25 veces se hacía cita de 1 a 5 autores y, dentro de ese rango, se contaron 54 citas. 
A partir de esta tabla, se puede observar una tendencia a realizar de entre 1 a 5 citas de otros investigadores por parte de los autores analizados y se ubicaron a 25 investigadores que tuvieron dicha práctica.

En el trabajo de Fuchs (1993), se presenta el concepto de dependencia mutua (mutual dependence) para explicar las posibilidades de descubrimientos científicos y los factores sociales que contribuyen a que así sea. La dependencia mutua es definida como el nivel de integración en un grupo o red de trabajo. Por ejemplo, la alta cocitación en documentos es considerada como un nivel alto de mutua dependencia, porque lo que ocurre en una parte de la red tiene consecuencias en otra, y viceversa.

La dependencia para la generación de conocimiento es indispensable, ya que en el intercambio se hace posible la contrastación y el debate. Además, cuando se revisa el trabajo de otros autores y se utiliza, es posible hacer un trabajo de verificación, así la confiabilidad y validez de un supuesto o de algunos resultados se pone a prueba, para reforzarlo o refutarlo, mucho más en los trabajos empíricos, pero también se permite la legitimación o consenso en trabajos de corte teórico. Cuando un autor se vuelve consistente, entonces se daría un caso de reforzamiento, hasta que probablemente, en la lógica de la ciencia, se rompa el paradigma.

\section{Consideraciones finales}

Es difícil señalar qué sería lo óptimo en cuanto a la cantidad de citas, ya que representa solo un aspecto cuantitativo que ofrece la panorámica de un comportamiento; no necesariamente más, significa mejor. Sin embargo, la ausencia de citar a otros sí puede representar un aislamiento en la producción científica y limitar la oxigenación de ideas.

Aunque en este estudio se presentó una panorámica muy general de la manera en que los investigadores del campo científico de la comunicación política interactúan por su citación, se presentan herramientas metodológicas que muestran elementos para comprender y reflexionar sobre su estructuración a partir de la citación. 
¿Y por qué podría resultar importante conocer la "cultura de citación" de los investigadores de un campo? Resulta pertinente en dos sentidos: el primero, debido al diálogo, la discusión o el debate que se propician para el choque de ideas y generación de conocimiento, al estilo filosófico de Nietzcsche ${ }^{15}$ que iría más orientado a la producción de conocimiento y al ejercicio científico; y el segundo, se presenta en el sentido de la capacidad de reproducir las prácticas en el ejercicio académico; la mayor parte de los investigadores transmiten sus prácticas a los alumnos con los que comparten el aula o incluso con practicantes, y la transferencia no solo se genera respecto del conocimiento, sino también de modelos de acción.

El análisis de las redes sociales permitió observar a partir de los datos un campo relativamente intrincado y activo en sus redes de citación, así como se pudo observar la existencia de una dinámica pluridisciplinar cuando se hace cita a autores externos al campo (Fuentes-Navarro, 1995).

Hasta este momento, quizá todavía exploratorio, se puede observar una estructuración relativamente sólida en las producciones y una tendencia de citación en investigadores con adscripción, sobre todo, a universidades públicas, como la Universidad de Guadalajara (Aceves, Sánchez Ruiz, Arredondo, Orozco, Sánchez Murillo e Ibarra) y la Universidad Nacional Autónoma de México (Delarbre, Vega y Byrd). También se pudo observar que el campo más cercano a la comunicación política podría ser el de las ciencias políticas por la importante presencia en la citación de investigadores de dicho campo (Segovia y Casanova).

Tener la percepción de los propios investigadores permite un acercamiento más claro a la manera en que se estructura el campo desde las prácticas subjetivadas, por lo cual se recomienda la triangulación de resultados como los presentados en este artículo, con la dimensión subjetiva.

Los resultados parecieran darle consistencia al argumento de que "la circulación del conocimiento científico depende menos del contenido

15 A la idea de que es a partir del encuentro de ideas que se produce el conocimiento. 
del mismo (dimensión simbólico-cognitiva) que de la red semiótico-material en la que el mismo está inserto" (Rodríguez, 2013, p. 9). Pero aún se requieren estudios y datos más exhaustivos para poder decir que se comporta de esta manera. Sin embargo, se propone que se empleen metodologías, como el análisis de redes sociales para su análisis y poder comprender la citación como un capital que circula para la constitución de un campo científico. A partir de lo expuesto, se puede concluir que se desarrolló una aproximación empírica de la investigación en comunicación política considerándolo como un campo en construcción.

\section{Referencias}

Aceves, F. (2002). De la construcción de las agendas a la centralidad mediática del espacio público: hallazgos y desafíos en la investigación de la comunicación política. Anuario de Investigación de la Comunicación, 9.

Ben-David,J. (1974). El papel de los científicos en la sociedad: un estudio comparativo. México: Trillas.

Bourdieu, P. (1997). Los usos sociales de la ciencia. Buenos Aires: INRA.

Bourdieu, P. (1999). Intelectuales, política y poder. Buenos Aires: Eudeba.

Bourdieu, P. (2000). La distinción: criterio y bases sociales del gusto. Madrid: Taurus.

Federico de la Rúa, A. de (2009). La perspectiva del interaccionismo estructural para el análisis de redes sociales. Redes: Revista Hispana para el Análisis de Redes Sociales, 17(12), 258-274.

Foucault, M. (1973). El orden del discurso. México: Tusquets.

Fuchs, S. (1993). A sociological theory of scientific change. Social Forces, 71(4), 933-953.

668 Las redes de citación como elemento de construcción del campo científico... - Alejandra Rodríguez-Estrada 
Fuentes-Navarro, R. (1998). La emergencia de un campo académico: continuidad utópica y estructuración científica de la investigación de la comunicación en México (Tesis de doctorado, Universidad de Guadalajara, Guadalajara, México).

Gaete, J. M. y Vásquez, J. I. (2008). Conocimiento y estructura en la investigación académica: una aproximación desde el análisis de redes sociales. Redes: Revista Hispana para el Análisis de Redes sociales, 14(5), 1-36.

Gil, J. y Ruiz, A. A. (2009). Análisis de las publicaciones de investigadores del subsistema de investigación científica de la UNAM 19812003. Redes: Revista Hispana para el Análisis de Redes Sociales, 17(1), 1-38.

Knorr-Cetina, K. D. (2005). La fabricación del conocimiento. Buenos Aires: Universidad Nacional de Quilmes.

Kuzhabekova, A. (2011). Impact of co-authorship strategies on research productivity: A social-network analysis of publications in russian cardiology (Tesis de doctorado, Universidad de Minesota, Estados Unidos).

Lazarsfeld, P., Berelson, B. y Gaudet, H. (1944). The peoples choice: How the voter makes up his mind in a presidential campaign. Columbia: Columbia University Press.

León, G. A. (2002). Teorías e investigación de la comunicación en América Latina: situación actual. Ámbitos, 8, 19-47.

Martínez, M. (2007). Agitación en el campo: nueve ideas para la investigación sobre comunicación política en España. Política y Sociedad, 44(2), 209-227.

Mattelart, A. (1997). Historia de las teorías de la comunicación. Madrid: Paidós. 
Moody, J. (2004). The structure of a social science collaboration network: Disciplinary cohesion from 1963 to 1999 . American Sociological Review, 69(2), 213-238.

Ochoa, O. (2000). Comunicación política y opinión pública. México: McGraw-Hill.

Ojeda, M. (2004). México antes y después de la alternancia política: un testimonio. México: El Colegio de México, Centro de Estudios Internacionales, Región y Sociedad.

Olivé, L. (1988). Conocimiento, sociedad y realidad: problemas del análisis del conocimiento y el realismo científico. México: Fondo de Cultura Económica.

Paláu-Cardona, S. (2009). Discursos y prácticas en el proceso de estructuración del campo académico de la comunicación en México: los investigadores de la dimensión política de los medios (Tesis de doctorado, Instituto Tecnológico y de Estudios Superiores de Occidente, Tlaquepaque, Jalisco, México).

Pérez, M. Á. (2006). Usos y abusos de la cienciometría. México: Centro de Investigación y de Estudios Avanzados.

Piedra, Y. (2010). Campo científico de la comunicación: examinando su estructura intelectual a través del análisis de cocitación. Revista Latina de Comunicación Social, 65, 204-213. Recuperado de http://www.redalyc.org/pdf/819/81915723015.pdf

Pineda de Alcázar, M. (2001). Las teorías clásicas de la comunicación: balance de sus aportes y limitaciones a la luz del siglo XXI. Opción, 1(32), 11-29.

Quatman, C. y Chelladruai, P. (2006). The social construction of knowledge in the field of sport management: A social network perspective 
(Tesis de doctorado, Ohio State University, Columbus, Ohio, Estados Unidos).

Ramírez, L. (2010). Campo científico y redes de coautoría en la psiquiatría: la producción científica psiquiátrica mexicana sobre el trastorno de la personalidad. Redes: Revista Hispana para el Análisis de Redes Sociales, 19(2), 20-39.

Ramos, C. (2012). Estructuras de comunicación en el campo de la ciencia social en Chile: un análisis de redes. Redes: Revista Hispana para el Análisis de Redes Sociales, 1(23), 7-42.

Reyes, M. C., O’ Quínn, J. A., Morales, J. M. y Rodríguez, E. (2011). Reflexiones sobre la comunicación política. Espacios Públicos, 14(30), 85-101. Recuperado de http://www.redalyc.org/articulo.oa?id=67618934007

Rodríguez-Estrada, A. (2015). Las prácticas y redes de investigadores del campo de la comunicación política en México (Tesis de doctorado, Instituto Tecnológico y de Estudios Superiores de Monterrey, Monterrey, México).

Rodríguez, L. (2013). Objetos subordinantes: la tecnología epistémica para producir centros y periferias. Revista Mexicana de Sociología, $75(1), 7-28$.

Rogers, E. M. (2003). Diffusion of innovations. Nueva York: The Free Press.

Romancini, R. (2006). O campo cientifico da Comunicao no Brasil, institucionalizacao e capital cienítico (Tesis de doctorado, Universidade de São Paulo, São Paulo, Brasil).

Russell, J. M., Madera Jaramillo, M. J. y Ainsworth, S. (2009). El análisis de redes en el estudio de la colaboración. Redes: Revista Hispana para el Análisis de Redes Sociales, 17, 39-47. 
Sogi, C., Perales, A., Anderson, A. y Bravo, E. (2002). Anales de la Facultad de Medicina. Production, 63, 191-200.

Van den Bulte, C. y Wuyts, S. (2007). Social networks and marketing. Massachusetts: Marketing Science Institute.

Wodak, R. (2003). De qué se trata el análisis crítico del discurso. En R. Wodak y M. Meyer, Métodos de análisis crítico del discurso (pp. 17-33). Barcelona: Gedisa. 